\title{
On the Origin of Resistive Switching Volatility in $\mathrm{TiO}_{2}$ thin films
}

\author{
Simone Cortese, ${ }^{1, \text { a) }}$ Maria Trapatseli, ${ }^{1}$ Ali Khiat, ${ }^{1}$ and Themistoklis Prodromakis ${ }^{1}$ \\ Nano Research Group, Electronics and Computer Science, University of Southampton, SO17 1BJ, \\ United Kingdom.
}

(Dated: 14 March 2016)

Resistive switching (RS) and Resistive Random Access Memories (ReRAMs) that exploit it have attracted huge interests for next generation non volatile memory (NVM) applications, also thought to be able to overcome flash memories limitations when arranged in crossbar arrays. A cornerstone of their potential success is that the RS between two different resistive states, usually High (HRS, High resistive state) and Low(LRS, Low Resistive State) is an intrinsic non-volatile phenomenon with the two states thermodynamically stable. Titanium Dioxide is one of the most common materials known to show non-volatile RS. In this paper we report the first observed volatile resistive switching (VRS) in a Titanium Dioxide thin film. The aim of this paper is to study and understand the VRS phenomenon to give an extensive picture of its underlying Physics. A possible exploitation of the VRS could be in access devices in ReRAM crossbar arrays.

PACS numbers: 81,85

Keywords: ReRAM, Selector Device, Access Device, Titanium Dioxide, Resistive Switching, Volatility, Device Physics.

\section{INTRODUCTION}

In the last decade memory technology has seen the rise of a new, potentially game-changer device for NVMs, the Memristor. This two terminal device, categorized as a fundamental circuital component together with Resistor, Capacitor and Inductor, has been postulated more than 40 years ago ${ }^{1}$. Yet, it stood from the fog of mere theory and it became reality just recently, when the resistive switching of a Titanium Dioxide thin film was for the first time related with memristor theory. ${ }^{2}$ The RS phenomenon consists in the reversible modification of the devices' internal resistance, switching between two, or more, stable resistive states. Devices that exploit RS phenomena are called ReRAMs and are now thought to be one of the best candidates to overcome FLASH limitations, due to their scalability and 3-D stacking capabilities when arranged in ordered arrays known as crossbars. The RS is indeed ideal for NVM applications: to switch between HRS and LRS the devices need to be driven just by voltage bias. When a device switches from HRS to LRS the process is usually known as SET operation whilst the opposite operation to restore the HRS is, on the contrary, known as RESET. The difference between SET and RESET could be in terms of voltage polarity (positive or negative) or different compliance current, depending on the device technology. ${ }^{3}$ The HRS are nonvolatile phenomena: HRS and LRS are two thermodynamically stable states of the same system and In order to induce a transition a voltage bias is required. This behaviour paved the way for their usage in NVM field.

From this original spark, the research boomed and many materials have been demonstrated to achieve a non-volatile RS, such as other oxides like Titanium

\footnotetext{
a) Electronic mail: simone.cortese@soton.ac.uk.
}

Dioxide, ${ }^{4}$ Hafnium Oxide ${ }^{5}$ Nickel Oxide $^{6}$, Tungsten Oxide $^{7}$, chalcogenides ${ }^{8}$ or oxide heterostructures.

Yet, it has been reported that resistive switching may also be volatile in few structures based on a vacuum (gap) layer between two electrodes ${ }^{9}$ or based on a $\mathrm{Cu} / \mathrm{TaO}_{2}$ stack $^{10}$ : the achieved LRS is not stable and automatically switches back to HRS without a RESET.

It is straightforward to conclude that a Volatile RS (VRS) is no longer useful for NVM applications, since every bit stored would be inherently self-cancelled by the volatility effect. Yet, it has been shown ${ }^{9}$ how the RS volatility could be used to solve a huge problem related with the NVM-usage of ReRAM crossbars: sneak currents. These currents rise during the crossbar readout process and are due to the combined effect of the ReRAM passive and bistable nature plus the shared bit and word line between different ReRAMs in the array that form alternative current paths with resistance lower or comparable to the targeted one, as can be seen from figure 1. The whole effect is that the readout process can be seriously compromised ${ }^{11}$.

To avoid sneak currents, an access devices or selector should be coupled with the ReRAM at each intersection between a word and a bit line in the array. Different examples of different selector systems have indeed been reported in literature. ${ }^{12-13-14-15-16-17}$ It is mandatory that the access device supplies enough non-linearity to the system to suppress sneak currents and that it must be compatible with crossbar fabrication. Hence, a filamentary switching device can be exploited as access device to tackle sneak currents due to its sharp turn on slope.

In this paper we present a VRS achieved with a 5 $\mathrm{nm}$ thick quasi-stoichiometric Titanium Dioxide thin film sandwiched within two nickel electrodes. The aim of this paper is to electrically characterize the film and the VRS in order to investigate the Physics of the phenomenon, so far unreported in the $\mathrm{TiO}_{2}$ films. 


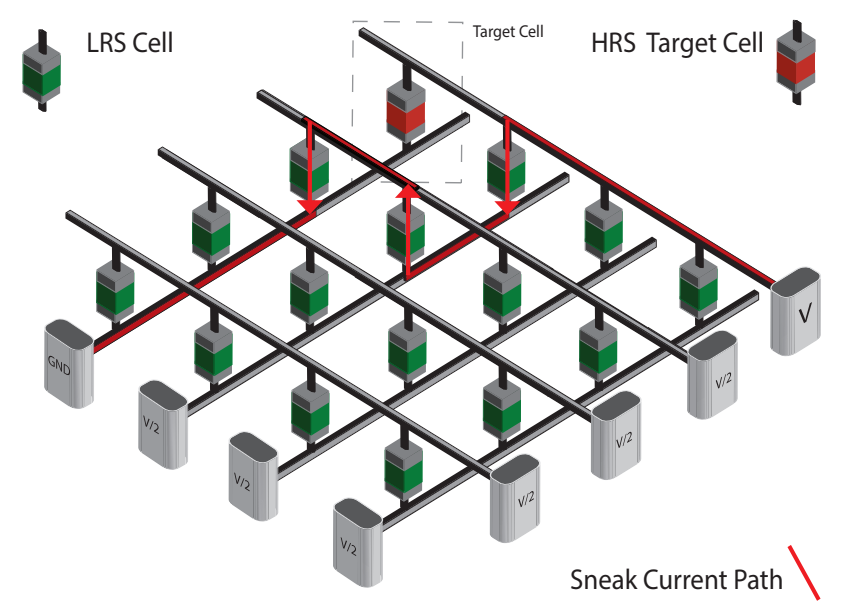

FIG. 1. Sneak current paths in ReRAM crossbar arrays through unselected cells. Current tends to flow through the lowest resistance path.

A model based to describe the volatile filamentary switching is also proposed and after a comparative analysis with other different mechanisms that could have given rise to a similar phenomena like Metal-Insulator Transitions (MIT). The possible exploitation of the VRS for ReRAM crossbar arrays access devices, due to the high non linearity of the phenomenon, is also briefly discussed.

\section{METHODS}

The symmetric metal-insulator-metal (MIM) structure investigated in this work was fabricated starting from a 6 -inch silicon wafer. A $200 \mathrm{~nm}$ thick layer of Silicon Dioxide was thermally grown via dry oxidation at $1000^{\circ}$. A $5 \mathrm{~nm}$ thick titanium layer as been deposited as adhesive layer for the subsequent $30 \mathrm{~nm}$ thick Nickel bottom electrode. Both adhesive and bottom electrode layers were deposited via e-beam evaporation. The $5-\mathrm{nm} \mathrm{TiO}_{2}$ active layer was deposited via $\mathrm{RF}$ sputtering using an AJA ORION sputterer from a 4-inch Ti target (purity $\geq 99.9 \%)$ in a Oxygen-Nitrogen rich environment (4.5 sccm oxygen flow and $10 \mathrm{sccm}$ nitrogen flow). Finally, a $30 \mathrm{~nm}$ thick Nickel Top Electrode was deposited again via e-beam evaporation. The layers were patterned via standard optical lithography. The lithography workflow followed this order for all the layers: spinning, exposure with negative tone masks, development and finally, material deposition. The devices sizes span from $1 \mathrm{x} 1$ to $60 \mathrm{x} 60 \mu \mathrm{m}^{2}$. The devices have a crossbar layout, as can be seen from figure 2 .

The oxidation states of the deposited $\mathrm{TiO}_{2}$ film have been studied via High-resolution XPS analysis. X-ray photoemission spectra were recorded by a Thermo Scientific Theta Probe Angle-Resolved X-Ray Photoelectron Spectrometer system. (a)

(b)

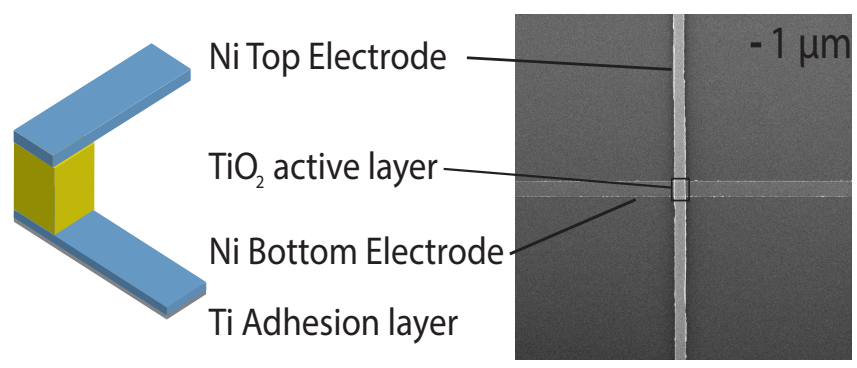

FIG. 2. (a) Volatile Resistive Switching Selector device stack, shown not in scale. (b) Scanning Electron Microscope image of a $1 \mathrm{x} 1 \mu \mathrm{m}^{2}$ selector device, showing the same layout as ReRAM crossbar arrays.

A monochromated Al K $\alpha$ X-ray source $(\mathrm{h} \nu=1486.6 \mathrm{eV})$ with a beam size of $400400 \mu \mathrm{m} 2$ was used, operating at $6.7 \mathrm{~mA}$ emission current, $15 \mathrm{kV}$ anode bias and $2 \cdot 10^{-9}$ mbar base pressure. Photoelectrons were collected over a cone of $\pm 30^{\circ}$. Data were collected with a pass energy of $200 \mathrm{eV}$ and $50 \mathrm{eV}$ for the survey and core level spectra, respectively. Survey and core level spectra were collected with $0.5 \mathrm{eV}$ and $0.02 \mathrm{eV}$ binding energy step, accordingly. The samples $\left(\mathrm{TiO}_{2-x}\right.$ thin films deposited on clean Si substrates), were inserted in the XPS chamber right after their deposition, to minimize the surface re-oxidation due to exposure in air and allow for more accurate measurements of the actual thin film chemical composition. The $\mathrm{C} 1 \mathrm{~s}$ peak has been always present on our thin films due to exposure in air and served as a convenient reference for charge shift correction. All data were analysed using the Avantage package. All the detected energies coincide with average reference energies from the NIST X-ray Photoelectron Spectroscopy Database. ${ }^{18}$ The electrical DC-IV characterization was carried out using a Keithley SCS4200 semiconductor device analyzer. Two tungsten needles were contacted with top and bottom electrodes of the MIM stack. One of the two electrodes was kept grounded, whilst the voltage bias was applied on the other.

Two configurations were tested: one configuration in which the bottom electrode was grounded and the voltage bias applied to the top electrode, labelled as configuration A. The opposite configuration, in which the top electrode was grounded and the bottom was biased, will be referred as configuration B throughout the paper. All the voltage sweeps were triangular, starting from 0 volt to $\mathrm{V}_{\text {stop }}$ and back to $0 \mathrm{~V}$ with two different $\dot{V}=d V / d t: \dot{V}$ $=2 \mathrm{Vs}^{-1}$ and $\dot{V}=2 \cdot 10^{-2} \mathrm{Vs}^{-1}$. These tests were made to evaluate the different response of the MIM system to different electric field variation rates. 

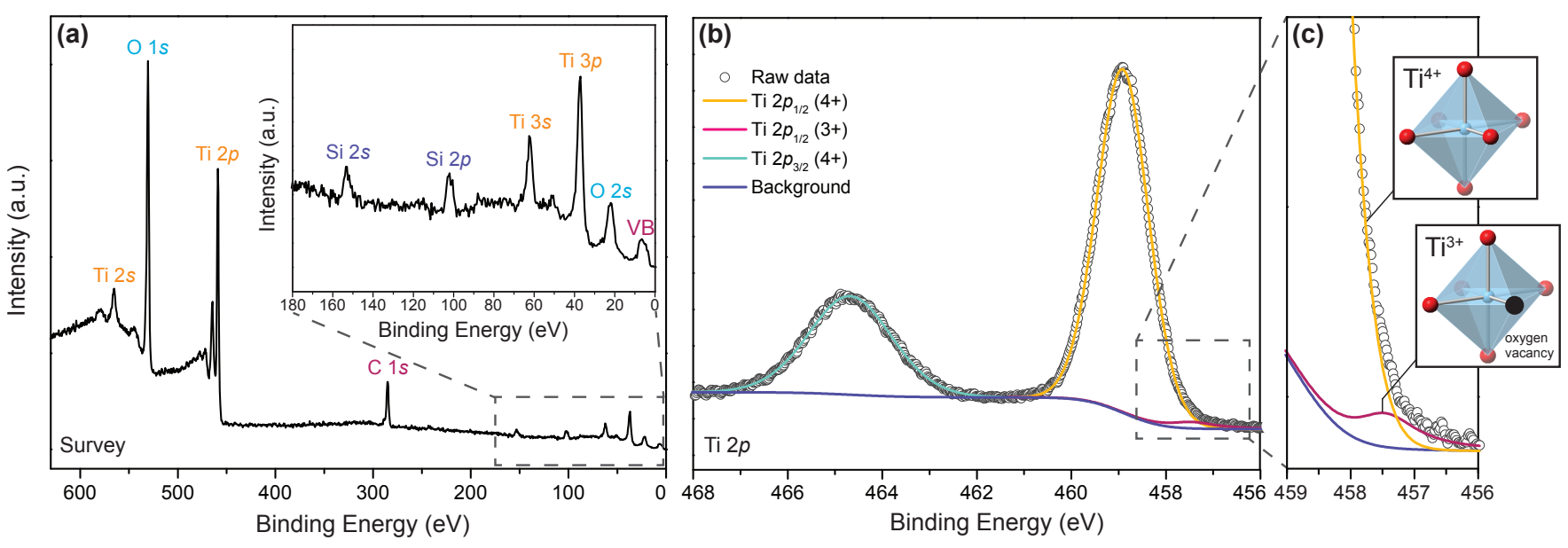

FIG. 3. XPS Characterization of the sputtered titanium dioxide $5 \mathrm{~nm}$ thick thin film.

\section{RESULTS}

\section{A. XPS characterization}

Figure 3-(a) portrays the survey spectrum of $\mathrm{TiO}_{2-x}$ thin film and all the resulting peaks from photoemission. O 1s, O 2p, Ti 2s, Ti 2p (doublet), Ti 3s and Ti 3p, peaks were present at the survey spectrum. $\mathrm{C}$ 1s was detected at $285.0 \mathrm{eV}$ and was used for charge shift correction. The occurrence of Si 2s and Si 2p peaks at 153.0 and 102.0 $\mathrm{eV}$ (shown in the inset) is associated with the $\mathrm{TiO}_{2-x}$ film thickness $(5 \mathrm{~nm})$ and the Si substrate. The valence band edge was detected in the binding energy range of $0-10 \mathrm{eV}$. Ti $2 \mathrm{p}$ core level spectrum is shown in detail in Figure 3 -(b). Its spin-orbit components, $\mathrm{p}_{1 / 2}$ and $\mathrm{p}_{3 / 2}$, at $458.9 \mathrm{eV}$ (yellow) and $464.7 \mathrm{eV}$ (cyan), respectively, were both of them associated with $\mathrm{Ti}^{4+}$ populations. Figure 3(c), reveals the lower binding energy area of the Ti $2 \mathrm{p}_{1 / 2}$ peak, at 459-456 eV. There, it can be seen more clearly, that the $\mathrm{p}_{1 / 2}$ component was fitted with an additional peak at $457.4 \mathrm{eV}$ (pink), which was associated with a very small population of $\mathrm{Ti}^{3+}$ ions and therefore oxygen vacancies. The octahedral coordination of Ti in Figure 3(c), is to give a generic visual representation of an oxygen vacancy next to a $\mathrm{Ti}^{3+}$ ion and it doesnt correspond to calculated bond angles and distances for this $\mathrm{TiO}_{2-x}$ thin film. The concentrations of $\mathrm{Ti}^{4+}$ and $\mathrm{Ti}^{3+}$ ions were calculated after appropriate peak fitting and they were 97.7 and $2.3 \%$ at., respectively. This result brings us to the conclusion that, our $\mathrm{TiO}_{2-x}$ thin film is very close to stoichiometry, with $\mathrm{x}$ being in the range of 0.03-0.05. Therefore we will refer to the film simply as $\mathrm{TiO}_{2}$ for the rest of the paper.

\section{B. Electrical Characterization}

The pristine resistance of all the $1 \mathrm{x} 1 \mu \mathrm{m}^{2}$ device tested, disregarding the configuration and $\dot{V}$ used, have shown a resistance always above

The Logaritmic Current-Voltage(IV) curve of a device under test can be seen in figure 4: the sweep was carried out in configuration A and with $\dot{V}=2 \mathrm{Vs}^{-1}$. The first sweep (shown as a dashed line) initially finds the device in its pristine HRS. The measured current is $20 \mathrm{pA}$, resulting in a resistance of $500 \mathrm{G} \Omega$. After this value, the current starts to rise with an exponential trend, suggesting a conduction mechanism related to Trap-Mediated Tunneling, Fowler-Nordheim emission or Schottky emission at the interface. At $-4.5 \mathrm{~V}$, the current spikes and the device switches to a LRS of $10 \mathrm{k} \Omega$. This LRS is kept till the voltage increases from $-5 \mathrm{~V}$ to $0 \mathrm{~V}$ in the last part of the triangular sweep. The subsequent sweep, independently of its polarity, finds the device again in its pristine like HRS without a previous RESET step of any kind. At negative polarities, the HRS is now kept till -2.5 V.50 G $\Omega$.

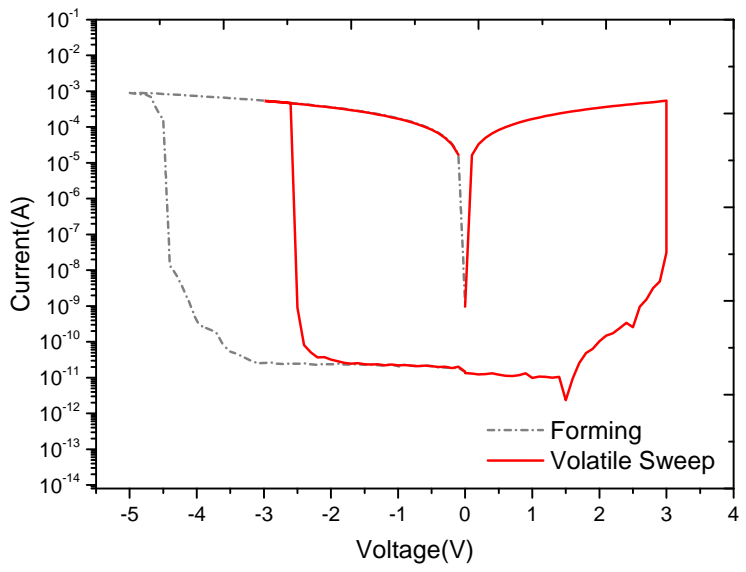

FIG. 4. IV characteristics curve of the $\mathrm{Ni}-\mathrm{TiO}_{2}$. The device self resets itself when the bias is removed, restoring the original HRS state. 

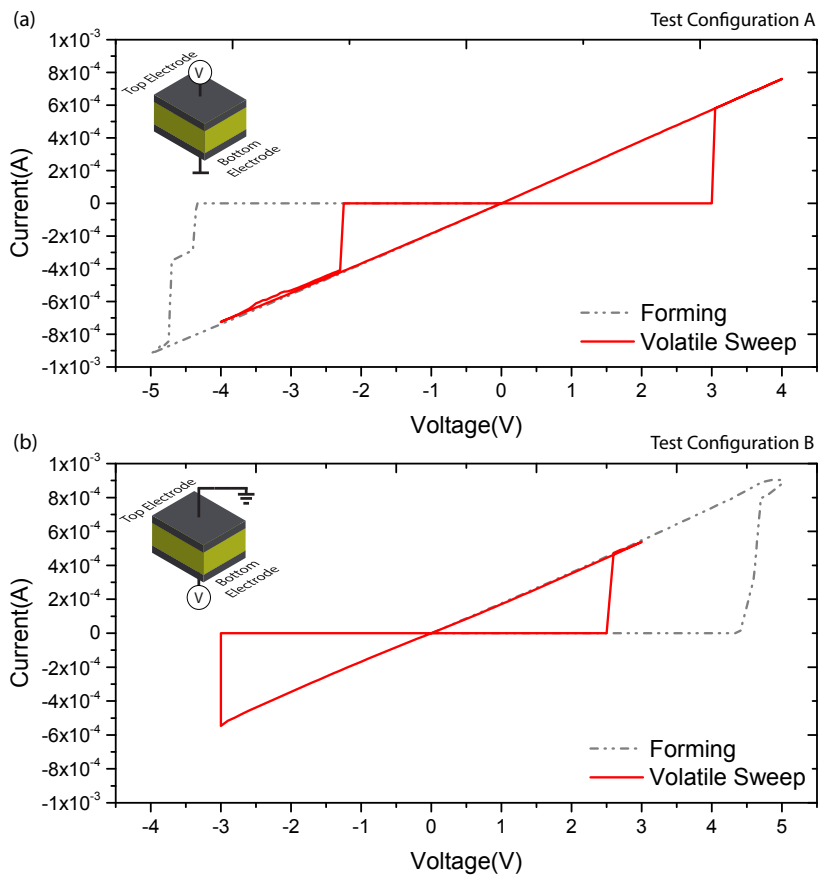

FIG. 5. Switching Top and Bottom electrode changed the polarity of the electroforming: IV comparison from test configuration A, shown in (a) and B, shown in (b).

Subsequently, a transition occurs, similarly to the first sweep. The achieved LRS has a resistance of $10 \mathrm{k} \Omega$ and it is exactly the same achieved previously. The same behaviour is obtained at positive polarities: the device starts from its HRS and switches at $3 \mathrm{~V}$ to the same LRS. The voltage thresholds for the switching are found to be generally lower (or higher, in case of negative polarities) after the first switching (e.g. from -4.5 of the first sweep to $-2.5 \mathrm{~V}$ for the subsequent sweep at $\mathrm{V}<0$ ): therefore, the first sweep will be referred as electroforming (or simply forming) sweep.

The LRS state is found to be linear $\left(\mathrm{R}^{2}>0.999\right)$ as can be seen from the LRS states of figure 5, suggesting a metal-like conduction at both polarities. It is finally of outstanding importance to remark that there is no necessity for a RESET sweep of any kind to restore the initial HRS since the HRS is spontaneously restored as soon as the voltage bias is removed.

Despite the symmetric MIM strucure, the electroforming has been reported just at negative polarities in test configuration A. In order to evaluate the effect of a different test configuration on the device behaviour, IV sweeps have been measured under test configuration $\mathrm{B}$, with the top electrode grounded and the bias applied on the bottom electrode. The results are shown in figure 5. Figure 5-(a) shows the linear IV characteristics under test configuration $\mathrm{A}$ : the device shows a negative forming and a volatile sweep, as described for figure 4 . Yet, when the

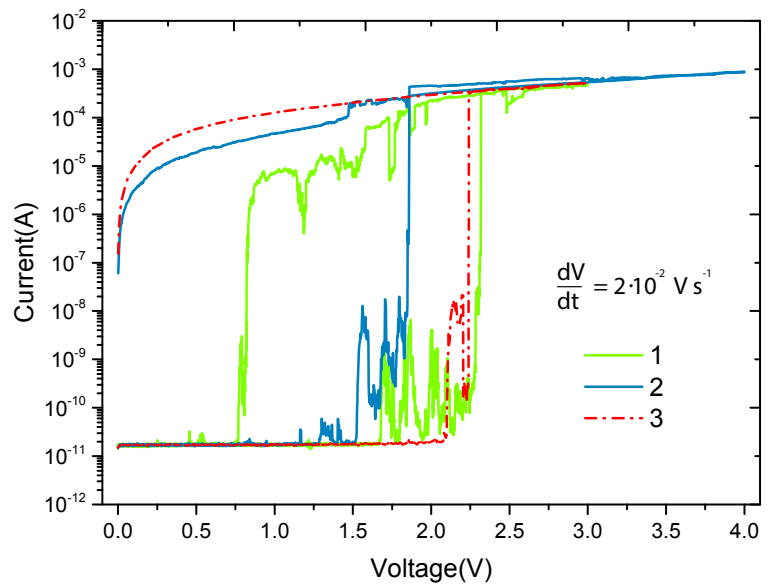

FIG. 6. Consecutive IV sweeps with $d V / d t=20 \mathrm{mV} / \mathrm{s}$ : the LRS stabilizes after the first collapse.

test configuration is switched from A to B, the electroforming is achieved at positive bias. The HRS and LRS are found in the $\mathrm{G} \Omega$ range and in a metallic-like $\mathrm{k} \Omega$ range, respectively. LRS is again the same at both polarities. This polarity dependent behaviour could imply that the switching is achieved close to a well defined interface of the film. To study the switching threshold dependency on the electric field variation, the $\dot{V}$ was decreased to $\dot{V}$ $=20 \mathrm{mV} / \mathrm{s}$ and the sweeps repeated on a new device. The voltage thresholds were shifted towards lower (or higher for negative biases) voltages, as can be seen from figure 6 . This effect is known to be related with different $\dot{V}$ sweeps. ${ }^{19}$ The initial resistive state is, once again, an HRS with currents below $20 \mathrm{pA}$, aligned with the values reported previously. Following the solid, green IV curve number 1 shown in figure, we can notice the current becomes unstable from $1.5 \mathrm{~V}$ to $2.4 \mathrm{~V}$, when the switching occurs. In this voltage region, the measured current oscillates between $20 \mathrm{pA}$ to a maximum of $10 \mathrm{nA}$. After the switching, a LRS of $7 \mathrm{k} \Omega$ is achieved. Yet, during the last part of the sweep, the IV starts to collapse and at $0.75 \mathrm{~V}$ the measured current falls back to the initial $20 \mathrm{pA}$ current baseline, showing a complete reset of the LRS. The subsequent IV curve, labelled as number 2 and shown in solid dark blue, initially finds the device in its HRS. As the previous IV, the current starts to oscillate before to reach the threshold voltage. After the switch, the LRS is again achieved. As the bias starts to decrease, at $1.5 \mathrm{~V}$ the IV shows a downward jump: the resistance changes from $7 \mathrm{k} \Omega$ to $20 \mathrm{k} \Omega$. Differently than before, the $20 \mathrm{k} \Omega$ state does not collapse but it is maintained until the bias is turned off. Any subsequent sweep, like the dashed number 3 curve, will find a switching from HRS to LRS with this latter maintained until the the voltage bias reaches $0 \mathrm{~V}$. The HRS must therefore be restored only after the voltage bias is removed. 

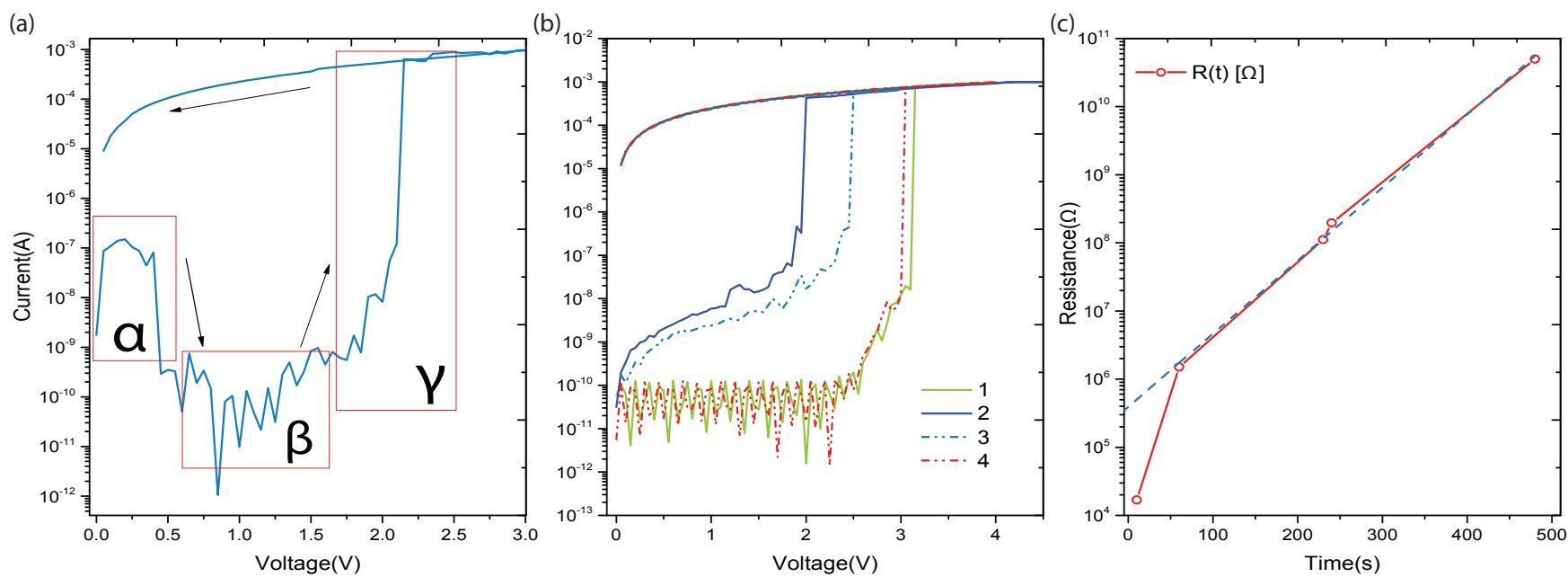

FIG. 7. (a) The initial resistive state in $\alpha$ collapses, before the device switches again. (b) Presence of unstable intermediate states is reported. (c) The device is able to self restore its initial HRS even when it undergoes into an apparent breakdown.

An intentional stress of 30 full, non-stop DC switching cycles stress tests were performed to the devices, in order to observe the evolution of the volatile behaviour and to evaluate its limits before a breakdown. It was possible to observe how, in some cases, the LRS started to become more robust: figure 7 -(a) shows how the switching phenomenon does not start from the pristine HRS, but it shows a shoulder between 0 and $0.5 \mathrm{~V}$, in the region labelled as $\alpha$. After $0.5 \mathrm{~V}$, the current decreases and enters the region $\beta$ in which the current is close to values for the pristine-like HRS. Subsequently, the device switches in region $\gamma$, maintaining the resistive state until the bias is removed. In figure 7-(b) the presence of intermediate resistive states after the stress test is shown. Initially, the device shows the same VRS described previously, as can be seen from curve 1. The following sweep, labelled as 2, starts from an HRS of $180 \mathrm{M} \Omega$ and switches to LRS at $2.1 \mathrm{~V}$. The successive light blue, dashed line sweep labelled as 3, starts from an HRS of $420 \mathrm{M} \Omega$ and switches to LRS at $2.8 \mathrm{~V}$. Finally, the red dashed 4th sweep starts from the original HRS state and switches to LRS at 3.1 $\mathrm{V}$, similarly to the curve 1 .

The breakdown of the device occurs when the LRS becomes not reversible: at this stage it is impossible a RESET of any kind, either self-made or induced by an applied bias. Yet, it has been observed that the HRS can be automatically restored by the devices after a certain time. Figure 7-(c) depicts the Resistance versus time curve. Before the breakdown and an apparently irreversible LRS, the device was showing a VRS similar to the ones previously described. The breakdown is declared when any of the subsequent sweeps immediately following each other shows that the LRS is maintained by the device, independently from the sweep maximum amplitude amplitude or polarity. Yet, after 60 seconds of pause within sweeps, the device shows that the resistance relaxed, increasing to $1 \mathrm{M} \Omega$. The trend continues till 500 $\mathrm{s}$ when the initial resistance is restored. The trend observed is exponential, as the linear fitting in logarithmic scale shows. The first resistance measured at 60 seconds seems to follow a faster the relaxation trend than the subsequent values reported. This time dependent relaxation of the resistive state is found to be present at both positive and negative polarities.

Another remarkable finding is that the application of a compliance current to the system does not affect extensively the switching behaviour, and the LRS achieved at the end of the sweep. As an example, a device formed at $-5.0 \mathrm{~V}$ with a Compliance Current (CC) of $100 \mu \mathrm{A}$ as achieved a LRS of $3.3 \mathrm{k} \Omega$. Instead, with a $\mathrm{CC}$ of $1 \mathrm{~mA}$, the device showed a LRS of $2.5 \mathrm{k} \Omega$, a value comparable with the previously reported $3.3 \mathrm{k} \Omega$.

\section{DISCUSSION}

The IV curves show devices in an initial HRS with currents of $20 \mathrm{pA}$ and a resulting resistance up to 500 G $\Omega$. Such an high resistance is explained by the high grade of stoichiometry of the sputtered titanium dioxide film, as discussed and shown in figure 3 . In $1 \times 1 \mu \mathrm{m}^{2}$ devices the current starts to rise before the switching with a conduction mechanism which could be linked to different oxide-related phenomena like Schottky emission, trap-assisted tunneling or Fowler-Nordheim, as also previously shown in a similar system ${ }^{20}$. At the threshold voltage, the transition is steep and, due to the rapid increase of current in the range of $5 \mathrm{mV} / \mathrm{dec}$, it can't be related to the previously cited oxide-based conduction mechanisms. These latter mechanisms are known to have their IV slopes co-strained by an intrinsic physical limit, known as sub threshold swing, that fixes the slope at a maximum value of $60 \mathrm{mV} / \mathrm{dec}^{21}$ The presence of a first 
sweep at higher voltage, the electroforming, is typical of a particular type of RS known as filamentary switching ${ }^{22}$ : its role is to form a metal-like filament shorting the electrodes, achieving the first RS. Any subsequent SET will be achieved at voltages lower than the forming voltage. In oxides, usually the filament is formed either by metal ions $^{23}$ or by oxygen ions, defects, vacancies drifting under the action of the external electric field ${ }^{24}$. Titanium Dioxide is known to be amongst the materials showing filamentary, oxygen vacancies based RS. ${ }^{4}$ The electroforming step can have the role of inducing the formation of the filament within the insulating oxide matrix. It is important to underline that the forming appears only at negative polarity, breaking the apparent MIM stack symmetry. The modification of the test configuration from $\mathrm{A}$ to $\mathrm{B}$ and the consequent variation in the electroforming, as shown in figure 2, implies that the forming step is strongly dependent on the electric field polarity.

The estimation of the Joule Heating energy available to the thin film system just before the switching results to be $1 \mathrm{pJ}$ or below. This value is too low to induce any significant increase of the temperature causing a thermaldriven switching ${ }^{25}$. For these reasons, we can conclude that the forming is electric field driven. The same considerations applies for all the SET phenomena subsequent to the forming.

The sweeps at the lowest $\dot{V}$ in figure 6 show how the device behaviour changes when the voltage variation rate is modified. As already discussed, this behaviour is related to the longer time available to the system to adapt to the electric field variation. The interesting feature shown by the slower sweeps is related to the collapse and subsequent stabilization of the LRS. The first shows a current collapse and a restoration of the initial HRS before to reach $0 \mathrm{~V}$, in a behaviour which could be thought similar to what has been reported elsewhere in materials showing a Metal-Insulator Transition (MIT) behaviour, like Vanadium Dioxide: ${ }^{26}$ this material has been long know to shows MIT, a phenomenon that switches a film from the original insulating HRS to a metal-like LRS with a steep increase in current ${ }^{27}$, similarly to what has been observed in the VRS devices. A typical fingerprint is the presence of an Hold Voltage Threshold : during a triangular sweep, the film returns to the original insulating HRS when it reaches this threshold, which is observed to be always smaller than the original switching threshold. Within this framework, it is straightforward to understand that the collapse of the first sweep could be related to a potential Hold Voltage due to a MIT-like phenomenon. Yet, the subsequent sweeps make clear that the VRS cannot be explained in terms of MITs. In the latter phenomenon, the presence of an Hold Voltage threshold in any sweep is very distinctive and always present. On the contrary, the VRS shows a stabilization of the LRS sweep after sweep, a phenomenon that is not compatible with MITs and, moreover, it is easily explainable with a filamentary option. Within this latter framework, the stabilization of the LRS can be related to the diameter growth of the conductive filament within the active layer. ${ }^{28}$ The intermediate $20 \mathrm{k} \Omega$ state reported in sweep 2 could be related to the partial shrinkage in filament radius that consequently decreases the filament cross section and therefore increases the overall resistance.

All these considerations corroborate the filamentary explanation for the VRS. Yet, it is known that the fingerprint of a filamentary switching mechanism is related to the independence of the current that the device supplies in respect to the device electrodes area. ${ }^{29}$ In figure 8 it is possible to observe the maximum current measured at $\mathrm{V}=-5$ volts and after the devices turned into their LRS (reversible for VRS in smallest devices and irreversible breakdown for larger area devices). The current measured is found to be constant at $5 \mathrm{~mA}$ and a resistance of $1 \mathrm{k} \Omega$ from $1 \mathrm{x} 1$ to $10 \mathrm{x} 10 \mu \mathrm{m}^{2}$ devices. The HRS has always showed currents of $20 \mathrm{pA}$ for all this group. The current density peaks in the smallest area and decreases subsequently. From $20 \mathrm{x} 20 \mu \mathrm{m}^{2}$ onwards, the maximum current slightly increases, reaching a maximum values of $20 \mathrm{~mA}$ in $60 \times 60 \mu \mathrm{m}^{2}$ devices.

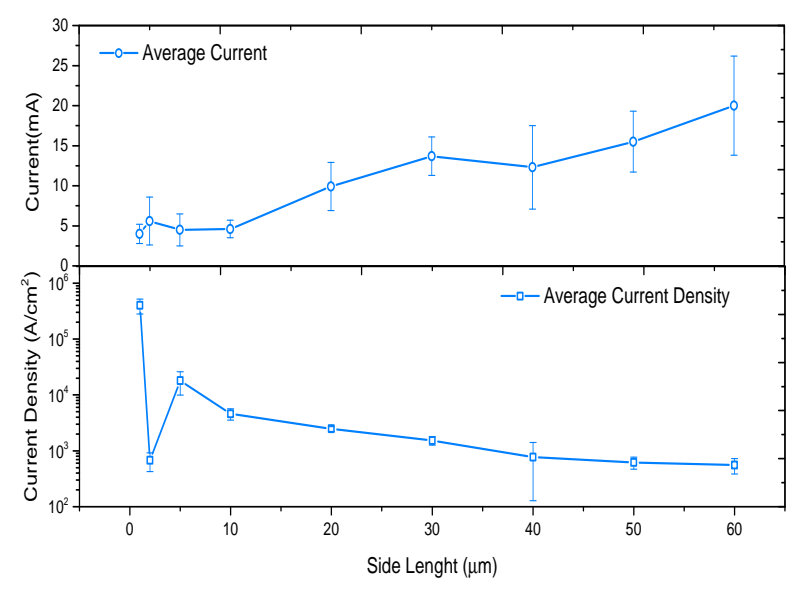

FIG. 8. Maximum current and maximum current density measured versus electrode side lenght.

Yet, the initial HRS was found to be more leaky with currents in the range of $100 \mathrm{pA}$. This is probably due to the presence of additional leakage currents trough oxide defects that increases the overall HRS current and may also slightly increase the maximum current measured. Decoupling the area-related sneak current contributions and the filamentary behaviour, we can conclude that smaller devices have their LRS independent by the electrode area up to $10 \times 10 \mu \mathrm{m}^{2}$, further corroborating the filamentary hypotesis.

But the filamentary hypothesis does not explain the unreported volatility observed. Figure 6 show how the initial pristine HRS is achieved before the end of the 
sweep, clearly indicating a volatile phenomenon: in addition, the LRS collapse before the end of the sweep and its successive stabilization could help to give an insight on the causes of volatility. The lack in thermal energy available suggests that the forming and the switching phenomena are electric field driven. From the XPS analysis, the film is known to be close to stoichiometry. Therefore is reasonable to assume that the density of the oxygen vacancies in the film is low. This could lead to a weaker filament and hence more subjected to spontaneous dissolution. The polarity dependent forming suggests that the positively charged oxygen vacancies move towards the top electrode, forming a filament with the tip moving towards the bottom electrode. After the filament formation, the resistance drops and the current surges. The current overshoot through the filament tip could lead to a joule heating: locally temperatures are known to increase easily till temperatures in the order of $1000 \mathrm{~K} .{ }^{30}$ This temperature could either reoxidize the tip or enhance oxygen vacancies diffusion. In the first option, it would be logical to expect that the reoxidation occurs in high current regime when more heath energy is available, inducing a RESET at higher voltages. Yet, it has been shown how the filament disruption occurs when the voltage bias is removed and no current flows in the system. It is therefore more likely that the thermal energy available remaining when the bias is turned off due to the local high increase of temperature enhances the vacancies diffusion and inducing a filament self-reset, before to be completely dissipated. The exponential trend in the resistance found in figure 7 -(c) shows how the values measured shift to the original values in a time width of 500 seconds. It is unlikely for the temperature to take such a long time to be dissipated. It is more likely that diffusion phenomena take place. Within this hypotesys, figure 7-(a) caught a filament during its dissolution.

Diffusion can be possible between the vacancies-rich filament and a thin interfacial layer between the titanium dioxide matrix and the nickel bottom electrode. The presence of a thin interfacial layer at the bottom electrode acting that helps self diffusion is an hypothesis that could explain why the electroforming is found only at negative polarities (in configuration A). The nature of the interfacial layer could be related to the exposure of the nickel bottom electrode to an oxygen plasma for several minutes, leading to a superficial oxidation. The plasma exposure has been much shorter in previously reported devices with similar structure and a 10 $\mathrm{nm}$ thin layer ${ }^{20}$ and can give an explanation why a similar behaviour has not been reported before. Moreover, a thin interfacial layer has already been associated to resistive switching volatility due to ions self diffusion in oxide systems. $^{10}$

The equation describing the net ion current under the action of an external electric field is stated as follows: ${ }^{31}$

$$
J_{D}=J_{\text {diffusion }}+J_{\text {Electric }}
$$

$\mathrm{J}_{\text {diffusion }}$ is the component of the ion current taking into account the movement of Ions due to diffusion while $\mathrm{J}_{\text {Electric }}$ the ion movement under the action of an external electric field. When the bias is applied, equation 1 stands and a dynamic equilibrium is reached between the two components. But when the bias is removed $\mathrm{J}_{\text {Electric }} \longrightarrow 0$ and diffusion takes over. Equation 1 becomes:

$$
J_{D}=J_{\text {diffusion }}
$$

Where $\mathrm{J}_{\text {diffusion }}$ is defined as follows:

$$
J_{\text {diffusion }}=-D \nabla n_{D}
$$

$\mathrm{D}$ is the diffusion cofficient for the ions, in our case oxygen vacancies, in the film and $n_{D}$ the vacancies density. The coefficient D is not constant, but it is defined as:

$$
D=D_{0} \exp \left[-\frac{E_{a}}{k_{b} T}\right]
$$

Where $\mathrm{E}_{a}$ is the activation energy for hopping and $\mathrm{T}$ the local temperature. For the VRS devices, after the bias is removed equation 1 is replaced by equation 2 where the diffusion coefficient depends on the local temperature, as shown in equation 4 . When the current surges the temperature peaks and the diffusion coefficient reaches its maximum values: this phenomenon, as a consequence, could allow a quick, thermal enhanced diffusion filament dissolution. When the temperature decreases, the diffusion continues but at a different rate. This model could explain why the self reset happens when the bias is removed and it is corroborated by the exponential trend in resistance versus time shown in figure 7-(c) and explain the faster resistance decrease rate observed for the value at 60 seconds in respect to the subsequent ones. In this case, the diffusion coefficient experienced could be higher, according to equation 4.

Therefore, the model we propose is based on an electric field driven filament formation with the presence of a thin nickel oxide interfacial layer. After the initial forming at negative polarity that forms the first filament, the electric field switches the devices connecting the electrodes by drifting the oxygen vacancies present in the film. The filament spontaneous dissolution is driven by thermal enhanced diffusion which could take place between the filament tip and an interfacial nickel oxide layer.

The VRS could be exploited in crossbar arrays as access devices. The structure and the fabrication is already compatible with current ReRAM manufacture and the materials involved are well known and BEOL compatible. One of the co-strain that the selector must stick to is the non linearity it can supply to the system, in order to be able to effectively suppress sneak currents. The VRS has shown to be able to achieve high non linearity, up to $10^{8}$, thanks to the filamentary mechanisms while most oxide-based selector devices are limited by the sub threshold swing coefficient. In addition, the filamentary switching allows to supply currents of up to $10 \mathrm{~mA}$ with a $1 \mathrm{x} 1 \mu \mathrm{m}^{2}$ device which is ideal to drive ReRAM without 
compromising crossbars' scalability. Therefore an application of the VRS as access device is very promising, although it requires additional electrical characterization which is not in the aim of this paper.

\section{CONCLUSION}

In this paper we have reported the very first volatile switching phenomenon achieved with a Titanium Dioxide $5 \mathrm{~nm}$ thick thin film. The switching is abrupt between a very high insulating state and a metallic state, supplying currents up to $10 \mathrm{~mA}$ in devices with $1 \mathrm{x} 1 \mu \mathrm{m}^{2}$ active area. The device Physics can be explained by the temporary formation of a metallic filament within the oxide layer under the action of an external electric field. When the bias is on, a dynamic equilibrium between electric field vacancies drift and thermal enhanced dissolution is reached. As soon as the electric field is removed the equilibrium is broken and the diffusion takes over. The possible presence of a nickel oxide layer could trigger oxygen vacancies diffusion from the filament's tip, making the restoration of the initial high resistive state easier. The high temperature that rises during the switching enhances the ion diffusion coefficient, further increasing the ion diffusion, achieving the filament dissolution. This mechanism could be exploited in access devices for ReRAM crossbar arrays due to its high non linearity, fabrication compatibility and scalability, although more research is required to evaluate its potential impact.

\section{ACKNOWLEDGEMENTS}

The authors acknowledge the financial support of EPSRC research grant EP K017829/1.

Data is available upon request at http://dx.doi.org/10.5258/SOTON/384819.

\section{REFERENCES}

${ }^{1}$ L. O. Chua, IEEE transactions on Circuit Theory, 1971, 18, year.

${ }^{2}$ D. Strukov, S. Snider, S. Stewart and S. Williams, Nature letters, 2009, 459, 180-83.

${ }^{3}$ R. Waser, R. Dittmann, G. Staikov and K. Szot, Advanced Materials, 2009, 21, 2632-2663.

${ }^{4}$ I. Salaoru, A. Khiat, Q. Li, R. Berdan and T. Prodromakis, Applied Physics Letters, 2013, 103, 233513.

${ }^{5}$ F. Nardi, S. Larentis, S. Balatti, D. Gilmer and D. Ielmini, IEEE transactions on Electron Device, 2012, 59, 2461-2467.

${ }^{6}$ S. Brivio, D. Perego, G. Tallarida, M. Bestetti, S. Franz and S. Spiga, Applied Physics Letters, 2013, 103, 153506.
${ }^{7}$ W. Chen, Y. Chen, E. Lau, Y. Lin, A. Chuang, Y. Yao, T. Chou, H. Lin, M. Lee, Y. Shih, Y. Hsieh and L. Chin-Yuan, Applied Physics A, 2011, 102, 901-907.

${ }^{8}$ D. Ielmini and Y. Zhang, Journal of Applied Physics, 2007, 102, 054517.

${ }^{9}$ C. Ho, H.-H. Huang, M.-T. Lee, C.-L. Hsu, T.-Y. Lai, W.-C. Chiu, M. Lee, T.-H. Chou, I. Yang, M.-C. Chen et al., Electron Devices Meeting (IEDM), 2012 IEEE International, 2012, pp. $2-8$.

${ }^{10}$ T. Liu, M. Verma, Y. Kang and M. Orlowski, Applied Physics Letters, 2012, 101, 073510.

${ }^{11}$ J. Zhou, K.-H. Kim and W. Lu, IEEE Transactions on Electron Devices, 2014, 61, 1369-1376.

${ }^{12}$ P. Woo Young, H. K. Gun, Y. S. Jun, M. K. Kyung, J. S. Seul, H. L. Min and S. H. Cheol, Electron Devices Meeting (IEDM), 2012 IEEE International, 2010, 19, year.

${ }^{13}$ N. Huby, G. Tallarida, M. Kutrzeba, S. Ferrari, E. Guziewicz, M. Godlewski et al., Microelectronic Engineering, 2008, 85, 2442-2444.

${ }^{14}$ R. S. Shenoy, G. W. Burr, K. Virwani, B. Jackson, A. Padilla, P. Narayanan, C. T. Rettner, R. M. Shelby, D. S. Bethune, K. V. Raman et al., Semiconductor Science and Technology, 2014, 29, 104005.

${ }^{15}$ S. H. Jo, T. Kumar, S. Narayanan, W. D. Lu and H. Nazarian, Electron Devices Meeting (IEDM), 2014 IEEE International, 2014, pp. 6-7.

${ }^{16}$ Y. Huang, R. Huang, Y. Cai, H. Wu, P. Yue, Y. Zhang, C. Chen and Y. Wang, Non-Volatile Memory Technology Symposium (NVMTS), 2012 12th Annual, 2012, pp. 85-87.

${ }^{17}$ J. Woo, D. Lee, E. Cha, S. Lee, S. Park and H. Hwang, Applied Physics Letters, 2013, 103, 202113.

${ }^{18}$ A. V. Naumkin, S. W. Kraut-Wass and C. J. Powell, http://srdata.nist.gov/xps/.

${ }^{19}$ J. P. Strachan, A. C. Torrezan, F. Miao, M. D. Pickett, J. J. Yang, W. Yi, G. Medeiros-Ribeiro and R. S. Williams, Electron Devices, IEEE Transactions on, 2013, 60, 2194-2202.

${ }^{20}$ S. Cortese, A. Khiat, D. Carta, M. E. Light and T. Prodromakis, Applied Physics Letters, 2016, 108, 033505.

${ }^{21}$ W. G. Vandenberghe, A. S. Verhulst, B. Sorée, W. Magnus, G. Groeseneken, Q. Smets, M. Heyns and M. V. Fischetti, $A p$ plied Physics Letters, 2013, 102, 013510.

${ }^{22}$ K. Tsunoda, Y. Fukuzumi, J. Jameson, Z. Wang, P. Griffin and Y. Nishi, Applied physics letters, 2007, 90, 113501.

${ }^{23}$ S. Gao, C. Chen, Z. Zhai, H. Liu, Y. Lin, S. Li, S. Lu, G. Wang, C. Song, F. Zeng et al., Applied Physics Letters, 2014, 105, 063504.

${ }^{24}$ R. Waser and M. Aono, Nature materials, 2007, 6, 833-840.

${ }^{25}$ L. Cario, C. Vaju, B. Corraze, V. Guiot and E. Janod, Advanced Materials, 2010, 22, 5193-5197.

${ }^{26}$ M. Son, J. Lee, J. Park, J. Shin, G. Choi, S. Jung, W. Lee, S. Kim, S. Park and H. Hwang, Electron Device Letters, IEEE, 2011, 32, 1579-1581.

${ }^{27}$ N. Mott, Reviews of Modern Physics, 1968, 40, 677.

${ }^{28} \mathrm{~S}$. Balatti, S. Larentis, D. Gilmer and D. Ielmini, Advanced materials, 2013, 25, 1474-1478.

${ }^{29}$ L. Courtade, C. Turquat, C. Muller, J. Lisoni and D. Wouters, Non-Volatile Memory Technology Symposium, 2007. NVMTS'07, 2007, pp. 1-4.

${ }^{30}$ D. Carta, A. Hitchcock, P. Guttmann, A. Regoutz, A. Khiat, A. Serb, I. Gupta and T. Prodromakis, Scientific reports, 2016, 6, year.

${ }^{31}$ S. Larentis, F. Nardi, S. Balatti, D. C. Gilmer and D. Ielmini, Electron Devices, IEEE Transactions on, 2012, 59, 2468-2475. 